\title{
Short-Term Solar Power Forecasting Using the Adaptive Network-Based Fuzzy Inference System
}

\author{
Wen-Yeau Chang \\ Department of Electrical Engineering \\ St. John's University \\ New Taipei City 25135, Taiwan \\ changwy@mail.sju.edu.tw
}

\author{
Ho-Chian Miao \\ Department of Electrical Engineering, \\ St. John's University \\ New Taipei City, Taiwan \\ 101m05017@stud.sju.edu.tw
}

\begin{abstract}
This paper proposes an adaptive network-based fuzzy inference system (ANFIS) based forecasting method for short-term solar power forecasting. An accurate forecasting method for power generation of the photovoltaic (PV) system is urgent needed under the relevant issues associated with the high penetration of solar power in the electricity system. To demonstrate the effectiveness of the proposed method, the method is tested on the practical information of solar power generation of a PV system installed on the St. John's University of Taiwan. Good agreements between the realistic values and forecasting values are obtained; the test results show the proposed forecasting method is accurate.
\end{abstract}

Keywords- solar power generation forecasting; photovoltaic system; adaptive network-based fuzzy inference system

\section{INTRODUCTION}

Rising crude oil prices highlights the exploitation of renewable energy applications. The solar power is one of the most attractive renewable energy technologies because of its low pollution. However, high penetration of solar power in the electricity system provides many challenges to the power system's operator, mainly due to the uncertainty of solar radiation.

Since the power produced by the PV system is varied with the solar radiation and the ambient temperature, unexpected variations of the PV system power generation may increase operating costs for the electricity system because the increased operation and maintain costs associated with cycling existing generation [1]. In addition, solar power forecasting plays an important role in the allocation of balancing power. Besides, solar power forecasting is used for the day-ahead scheduling of conventional power plants and trading of electricity on the spot market [2].

Recently, several methods have been employed for the solar power forecasting. The solar power forecasting methods can be generally categorized into three groups, physical methods, statistical methods and artificial intelligence methods.

Physical systems use parameterizations based on a detailed physical description of the atmosphere, to reach the best prediction precision. Usually, solar radiation given by the weather service on a coarse grid is transformed to the onsite conditions at the location of the PV system.
Physical methods have advantages in long-term solar power prediction. Several physical methods have been developed based on using weather data with sophisticated meteorological for solar radiation forecasting and solar power predictions [1].

Statistical methods aim at finding the relationship of the on-line measured power data. For a statistical model, the historical data of the PV system may be used. Statistical models are easy to model and cheaper to develop compared to other models. The statistical method has advantages in short-term solar power prediction. The disadvantage with this method is that the prediction error increases as the prediction time increases. The main methods of statistical methods are time series based methods [3]. Time series based methods include the auto regressive (AR), AR with exogenous input (ARX), auto regressive moving average (ARMA), and auto regressive integrated moving average (ARIMA).

Recently, with the development of artificial intelligence, various artificial intelligence methods for solar power prediction have been developed. The new developed methods include artificial neural network [4], fuzzy logic methods [5], weighted support vector machine [6], and grey model [7]. The artificial intelligence methods are self-designing ones that can be automatically adjusted in changing system.

To increase the accuracy of short-term solar power forecasting this paper takes the ANFIS based method to account for the nonlinearity and periodicity in the solar power generation time series. This paper deals with the power generation forecasting of PV system and is divided into five sections. After a brief introduction, Section II introduces the adaptive network-based fuzzy inference system. Section III describes the he ANSIF based solar power forecasting method. Numerical results are described in Section IV. The conclusions of the paper are summarized in Section V.

\section{ADAPTIVE NETWORK-BASED FUZZY INFERENCE SYSTEM}

Fuzzy logic is based on modeling human thinking and perception. Fuzzy systems make up the rule base and estimate sampled functions from linguistic input to linguistic output [8]. The neural networks are the powerful tool of artificial intelligence. The neural networks were 
widely applied in optimization, pattern recognition, forecasting. The main property of neural network is that it can learn from examples is the fact of preferring neural network in nonlinear or complex problems [9]. ANFIS was proposed via taking full advantage of both the processing fuzzy information of the fuzzy system and self-learning of the neural network.

Jang proposed the ANFIS model in 1993 [10]. ANFIS is based on a special fuzzy inference structure: the TakagiSugeno model and its output is a linear combination of all input variables [11]. In ANFIS model, the input series are converted to fuzzy inputs by using membership function for each input series. The membership function can be any shape but it depends on the data set [12].

ANFIS has been applied in several forecasting domains such as electricity price forecasting [11], weather forecasting [12], solar radiation data forecasting [13], daily stream flow forecasting [14], internet traffic time series forecasting [15] and demand forecasting [16]. In this paper, the membership function and fuzzy rule is obtained by historical solar power generation.

The typical structure of ANFIS is shown as Figure 1. ANFIS contain layers: the fuzzy layer, the product layer, the normalized layer, the defuzzification layer, and the output layer. Every node at the same layer has similar function. The framework of ANFIS can be expressed as following:

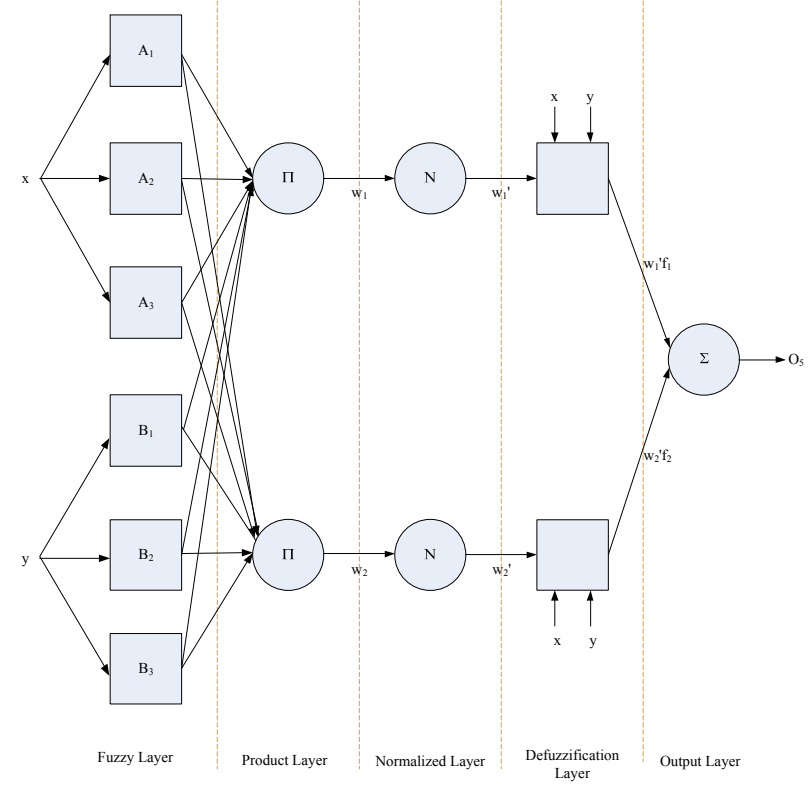

Figure 1. The typical structure of ANFIS.

The first layer is the fuzzy layer. The fuzzy layer contains of adaptive nodes that generate the membership grades of linguistic labels. Any appropriate parameterized membership function can be used such as the generalized bell function. $A_{1}, A_{2}, A_{3}, B_{1}, B_{2}, B_{3}$ are the linguistic labels used in the fuzzy set for dividing the membership functions. The relationship between the output and input functions of this layer can be expressed as below:

$$
O_{1, i}=\mu_{A i}(x), \quad i=1,2,3
$$

where $O_{1, i}$ is output function, and $\mu_{A i}$ is the membership function of the fuzzy set A.
The second layer is the product layer. The product layer consists of rule nodes designated as $\Pi$ which signifies the firing strength of each rule. The output of the product layer is the product of the input signal, which is defined as follows:

$$
O_{2, i}=w_{i}=\mu_{A i}(x) \times \mu_{B i}(y), \quad i=1,2,3
$$

where $O_{2, i}$ is output of the product layer, $\mu_{A i}$ is the membership function of the fuzzy set $A$, and $\mu_{B i}$ is the membership function of the fuzzy set B.

The third layer is the normalized layer. In this layer the fixed nodes are labeled as N. The output of the normalized layer is to normalize the weight function or the sum of all the rules firing strength as following:

$$
O_{3, i}=w_{i}^{\prime}=\frac{w_{i}}{w_{1}+w_{2}+w_{3}}, \quad i=1,2,3
$$

where $O_{3, i}$ is output of the normalized layer, $w_{i}$ is output of the product layer.

The fourth layer is the defuzzification layer. The nodes in the defuzzification layer are also adaptive nodes besides the nodes in the fuzzy layer. Those adaptive nodes calculate the rule outputs based on consequent parameters. The adaptive nodes of this layer calculate the rule outputs based on consequent parameters by following equation:

$$
O_{4, i}=w_{i}{ }^{\prime} f_{i}=w_{i}{ }^{\prime}\left(p_{i} x+q_{i} y+r_{i}\right), \quad i=1,2,3
$$

where $O_{4, i}$ is output of the defuzzification layer and $p_{i}, q_{i}, r_{i}$ are consequent parameters of the node.

The fifth layer is the output layer. This layer is the final layer. The output layer gives the output by the summation of all incoming signals. The fixed node in this layer is labeled as $\Sigma$ calculates the overall output from the sum of the node input signals. The output of the output layer can be expressed as below:

$$
O_{5, i}=\sum_{i=1}^{3} w_{i}{ }^{\prime} f_{i}, \quad i=1,2,3
$$

where $O_{5, i}$ is the output of the fifth layer which is the academic marks, $w_{i}$ is output of the defuzzification layer.

The training scheme of the ANFIS is a two-pass process over a number of epochs. The node outputs of first layer to the fourth layer are calculated through each epoch. At the fifth layer, the consequent parameters are calculated using a least-squares regression method [13]. During each epoch, the output of the ANFIS is calculated and after calculation of the error, the ratio of error is back propagated over every layers and those values are adapted based on error descent gradient method [14].

\section{ANSIF BASED SOLAR FORECASTING METHOD}

The architecture of the ANSIF based solar power forecasting method is shown in Fig. 2. The ANSIF model was developed for $10 \mathrm{~min}$. ahead solar power forecasting. The architecture of ANSIF used in this study contains five layers. The first layer (fuzzy layer) has 4 inputs: $x$ for the solar power output of 20 minutes ago, y for the solar power output of 10 minutes ago, $\mathrm{z}$ for the current solar power output, and $\mathrm{u}$ for the current solar radiation value. The output layer has one output for the 10 minutes ahead solar power forecasting. The number of fuzzy rules is 81 . The membership functions of the ANSIF model are the generalized bell functions. 


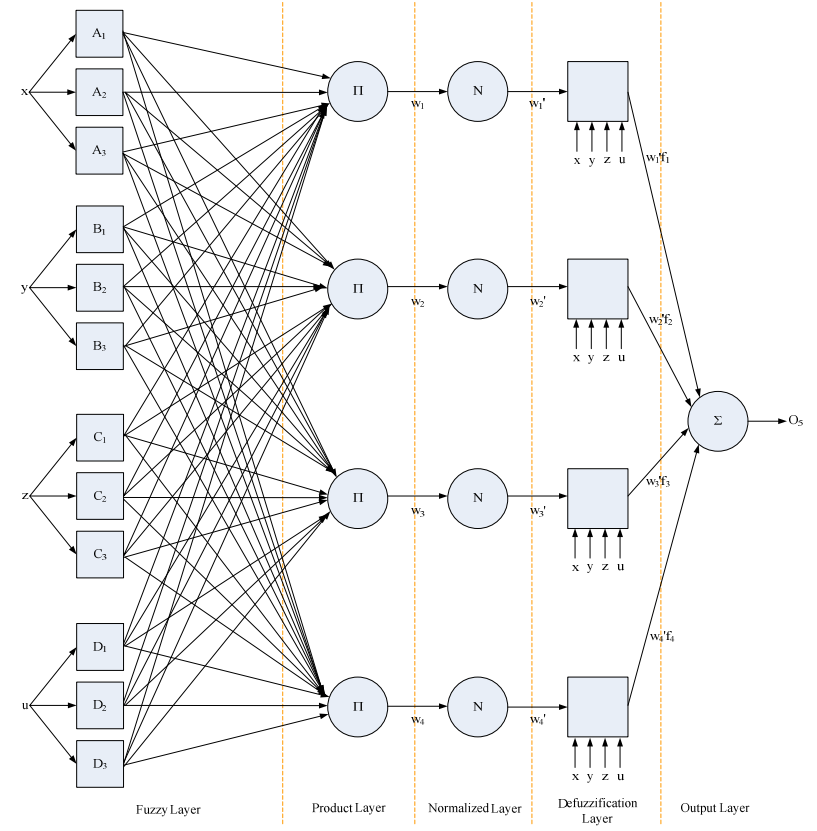

Figure 2. The architecture of the ANSIF based solar power forecasting method.

\section{NUMERICAL RESULTS}

In order to verify the proposed forecasting method, the method was used for solar power forecasting in Taiwan. Solar power forecasting was computed using the historical solar power and solar radiation data for every 10 minutes for a 9kW PV system installed in St. John's University of Taiwan. The solar power time series data for this PV system were recorded. In order to ensure a clear comparison, no exogenous variables are considered. Because of seasonal atmospheric weather characteristics, the solar power and solar radiation data are divided into 4 categories: spring, summer, autumn and winter. The four season day test data results are shown below.

From the winter day data, the following days were selected: January $21-25,2015$, corresponding to a typical winter day. The historical data set with 720 patterns were divided into a training data set for the ANFIS, of 576 patterns, collected from January 21-24, and a test data set of 144 patterns, collected from January 25. Numerical result of the ANFIS-based method for typical winter day is shown in Fig. 3.

From the spring day data, the following days were selected: March 21-25, 2015, corresponding to a typical winter day. The historical data set with 720 patterns were divided into a training data set for the ANFIS, of 576 patterns, collected from March 21-24, and a test data set of 144 patterns, collected from March 25. The numerical results of the ANFIS-based method for typical spring day are shown in Fig. 4.

From the summer day data, the following days were selected: August 21-25, 2014, corresponding to a typical winter day. The historical data set with 720 patterns were divided into a training data set for the ANFIS, of 576 patterns, collected from August 21-24, and a test data set of 144 patterns, collected from August 25. The numerical results of the ANFIS-based method for typical summer day are shown in Fig. 5.

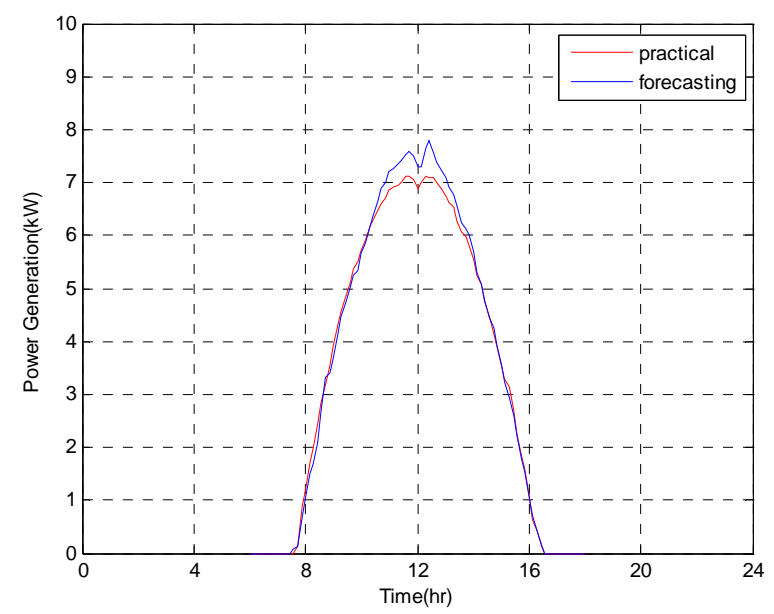

Figure 3. Numerical result of the ANFIS-based method for typical winter day.

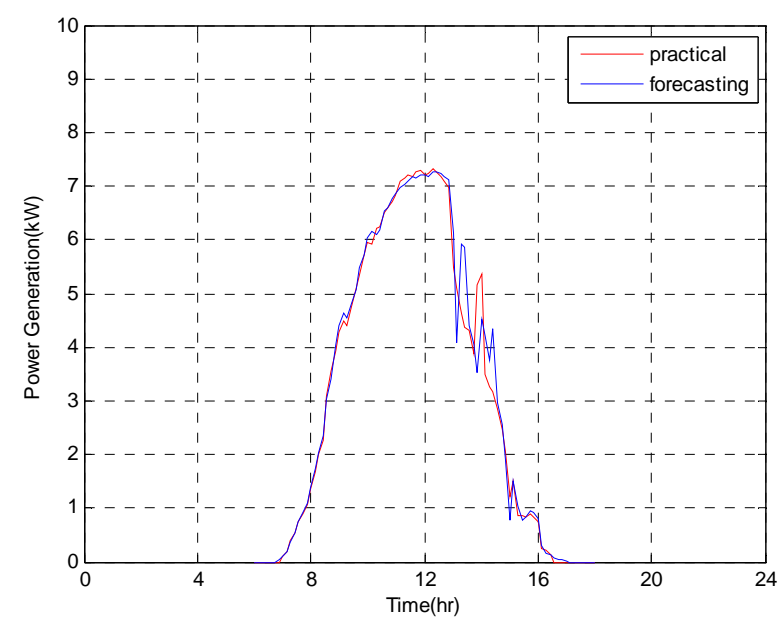

Figure 4. Numerical result of the ANFIS-based method for typical spring day.

From the autumn day data, the following days were selected: October 14-18, 2014, corresponding to a typical winter day. The historical data set with 720 patterns were divided into a training data set for the ANFIS, of 576 patterns, collected from October 14-17, and a test data set of 144 patterns, collected from October 18. The numerical results of the ANFIS-based method for typical summer day are shown in Fig. 6.

Comparing the four season data, a general conclusion that may be drawn from the obtained results is that proposed forecasting methods can forecast the solar power accurately.

\section{CONCLUSIONS}

This paper proposes an ANFIS based forecasting method using for $10 \mathrm{~min}$. ahead solar power forecasting. The performance of the proposed method to short-term solar power forecasting is effective. An evaluation of the forecast methods is performed, using the practical information of solar power generation of a PV system. The results demonstrate the effectiveness of the proposed 
forecasting method and this method provided improved accuracy in the solar power forecasting.

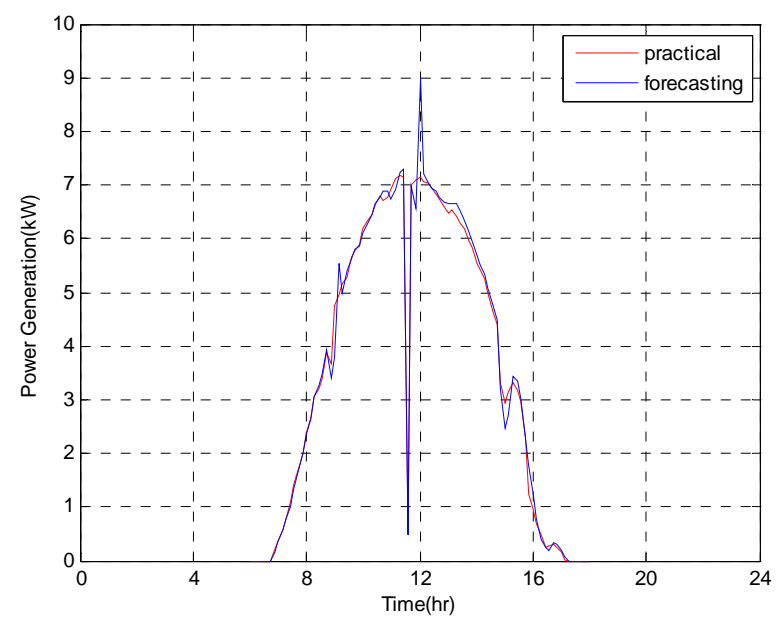

Figure 5. Numerical result of the ANFIS-based method for typical summer day.

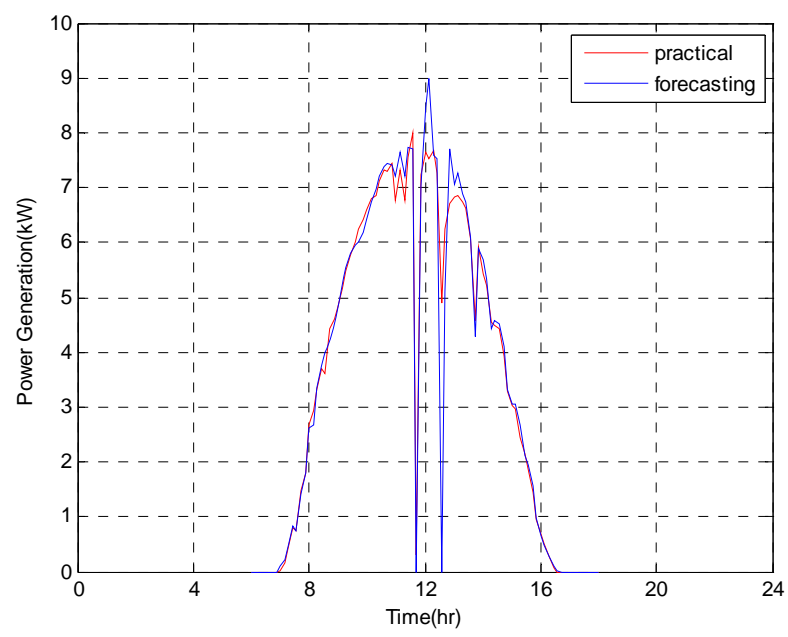

Figure 6. Numerical result of the ANFIS-based method for typical winter day.

\section{ACKNOWLEDGMENT}

The authors would like to express their acknowledgements to the Ministry of Science and Technology of ROC for the financial support under Grant MOST 103-2221-E-129 -011.

\section{REFERENCES}

[1] J. Bing, O. Bartholomy, and P. Krishnani, "Validation of Solar PV Power Forecasting Methods for High Penetration Grid Integration," Proc. 2012 IEEE Power and Energy Society General
Meeting, IEEE Press, July 2012, pp. 1-6, doi: 10.1109/PESGM. 2012.6345562.

[2] W.Y. Chang, "Comparison of three short term photovoltaic system power generation forecasting methods," Applied Mechanics and Materials, vol. 479-480, pp. 585-589, Jan. 2014.

[3] P. Bacher, H. Madsen, and H.A, Nielsen, "Online short term solar power forecasting," Solar Energy, vol. 83, pp. 1772-1783, 2009.

[4] W.Y. Chang, "Power generation forecasting of solar photovoltaic system using radial basis function neural network," Applied Mechanics and Materials, vol. 368-370, pp. 1262-1265, Aug. 2013.

[5] E. D'Andrea and B. Lazzerini, "Fuzzy Forecasting of Energy Production in Solar Photovoltaic Installations," Proc. 2012 IEEE International Conference on Fuzzy Systems (FUZZ-IEEE), IEEE Press, June 2012, pp. 1-8, doi: 10.1109/FUZZ-IEEE.2012.6251161.

[6] R. Xu, H. Chen, and X. Sun, "Short-Term Photovoltaic Power Forecasting with Weighted Support Vector Machine," Proc. 2012 IEEE International Conference on Automation and Logistics (ICAL), IEEE Press, Aug. 2012, pp. 248 - 253, doi: 10.1109/ICAL.2012.6308206.

[7] Y.Z. Li, R. Luan, and J.C. Niu, "Forecast of Power Generation for Grid-Connected Photovoltaic System Based on Grey Model and Markov Chain," Proc. 3rd IEEE Conference on Industrial Electronics and Applications (ICIEA 2008), IEEE Press, June 2008, pp. 1729- 1733, doi: 10.1109/ICIEA.2008.4582816.

[8] H.K. Kwan and Y. Cai, "A fuzzy neural network and its application to pattern recognition," IEEE Trans. Fuzzy Systems, vol. 2, no. 3, pp. 185-193, Aug. 1994.

[9] W.Y. Chang, "Application of back propagation neural network for partial discharge pattern recognition," Applied Mechanics and Materials, vol. 577, pp. 511-514, June 2014.

[10] J.S.R. Jang, "ANFIS: adaptive-network-based fuzzy inference system," IEEE Trans. Systems, Man and Cybern., vol. 23, no. 3, pp. 665-685, May 1993.

[11] H. Zhou, X.H. Wu, and X.G. Li, "An ANFIS Model of Electricity Price Forecasting Based on Subtractive Clustering," Proc. 2011 IEEE Power and Energy Society General Meeting, IEEE Press, July 2011, pp. 1-5, doi: 10.1109/PES.2011.6039228.

[12] M. Rahman, A.H.M.S. Islam, S.Y.M. Nadvi, and R.M. Rahman, "Comparative Study of ANFIS and ARIMA Model for Weather Forecasting in Dhaka," Proc. 2013 International Conference on Informatics, Electronics \& Vision (ICIEV), IEEE Press, May 2013, pp. 1-6, doi: 10.1109/ICIEV.2013.6572587.

[13] A. Mellit, A. Hadjarab, N. Khorissi, and H. Salhi, "An ANFISBased Forecasting for Solar Radiation Data from Sunshine Duration and Ambient Temperature," Proc. 2007 IEEE Power Engineering Society General Meeting, IEEE Press, June 2007, pp 1-6, doi: 10.1109/PES.2007.386131.

[14] E. Khadangi, H.R. Madvar, and M.M. Ebadzadeh, "Comparison of ANFIS and RBF Models in Daily Stream Flow Forecasting," Proc. 2nd International Conference on Computer, Control and Communication (IC4 2009), IEEE Press, Feb. 2009, pp. 1-6, doi: 10.1109/IC4.2009.4909240.

[15] S. Chabaa, A. Zeroual, and J. Antari, "ANFIS Method for Forecasting Internet Traffic Time Series," Proc. 2009 Mediterrannean Microwave Symposium (MMS), IEEE Press, Nov. 2009, pp. 1-4, doi: 10.1109/MMS.2009.5409834.

[16] S. Mohammadi, H. Keivani, M. Bakhshi, A. Mohammadi, M.R. Askari, and F. Kavehnia, "Demand Forecasting Using Time Series Modelling and ANFIS Estimator," Proc. 41st International Universities Power Engineering Conference (UPEC'06), IEEE Press, Sept. 2006, vol. 1, pp. 333-337, doi: 10.1109/UPEC. 2006.367770 . 